International Journal of Modern Physics B

Vol. 23, Nos 20 \& 21 (2009) 4414-4421

(C) World Scientific Publishing Company

\title{
RPA APPROACH TO NON-LINEAR TRANSPORT IN QUANTUM DOTS
}

\author{
B. TANATAR \\ Department of Physics, Bilkent University, 06800, Ankara, Turkey \\ tanatar@fen.bilkent.edu.tr
}

V. MOLDOVEANU

National Institute of Materials Physics, P.O. Box MG-7, 077125 Bucharest-Magurele, Romania valim@celsius.infim.ro

Received 24 July 2009

\begin{abstract}
An accurate theoretical treatment of electron-electron interactions in mesoscopic systems is available in very few cases and approximation schemes are developed in most of the applications, especially for many-level quantum dots. Here we present transport calculations within the random-phase approximation for the Coulomb interaction using the Keldysh Green's functions formalism. We describe the quantum dot systems by a tight-binding Hamiltonian. Our method is similar to the one used by Faleev and Stockman [Phys. Rev. B 66085318 (2002)] in their study of the equilibrium properties of a homogeneous 2D electron gas. The important extension at the formal level is that we combine the RPA and the Keldysh formalism for studying non-linear transport properties of open quantum dots. Within the Keldysh formalism the polarization operator becomes a contour-ordered quantity that should be computed either from the non-interacting Green functions of the coupled quantum dot (the so-called $G_{0} W$ approximation) either self-consistently ( $G W$ approximation). We performed both non-selfconsistent and self-consistent calculations and compare the results. In particular we recover the Coulomb diamonds for interacting quantum dots and we discuss the charge sensing effects in parallel quantum dots.
\end{abstract}

Keywords: Coulomb blockade; Quantum dots; Nonlinear transport.

\section{Introduction}

Quantum dot systems are commonly used in transport experiments in order to explore various effects coexisting at nanoscale: sequential tunneling, coherence or Coulomb blockade (see Ref. 1 for a review). At the theoretical level the main problem is to handle a quantum transport formalism that includes and describes as much as possible from the many-body physics of the electrons passing through a quantum dot.

The non-linear transport properties of interacting or non-interacting quantum dot systems have been studied mostly within the non-equilibrium Keldysh Green's function formalism. ${ }^{2}$ The steady-state current was calculated for small quantum 
dots with a spin-degenerate level and the interacting Green function is usually obtained via the equation of motion method (see e.g. Ref. 3 and the references therein). Some time ago Henrickson et al. ${ }^{4}$ took the challenge of implementing the GreenKeldysh formalism for few-level quantum dots using a selfconsistent Hartree approximation (SCHA) for the interaction self-energy. This mean-field approach provided interesting results in the Coulomb blockade regime and allowed for a discussion of the I-V curves.

Nevertheless, in the context of controlled dephasing in mesoscopic interferometers Coulomb-coupled to charge a detector ${ }^{5}$ one is obliged to include inelastic electron-electron scattering processes in the calculations and therefore to go beyond the mean-field approximation. A first attempt to compute the dephasing rate of a quantum dot coupled to a quantum point contact was done by Silva and Levit ${ }^{6}$; the authors computed the interaction self-energy up to the second order in the interaction strength. Later on the ring-dot geometry of the experiment ${ }^{5}$ was theoretically considered in Ref. 7 along the same lines. In order to treat on equal footing the interdot and intradot interactions we have recently developed a non-selfconsistent random-phase approximation for computing the Keldysh-Green functions. ${ }^{8}$ The method was used in the study of T-shaped interferometers and clearly emphasized the role of the intradot interaction in dephasing.

In the present work we improved the RPA method by including a selfconsistent procedure in computing the polarization operator. In the highly non-linear regime the self-consistent scheme guarantees the current conservation (see Thyegesen et al. ${ }^{9}$ ). As an application of this method we obtain the Coulomb diamonds of a single quantum dot and the charge sensing properties of two parallel quantum dots.

The rest od this paper is organized as follows. Section 2 provides the model we use to treat open quantum systems and theoretical background for the Keldysh formalism. Numerical results for a number of quantum dot systems are presented in Section 3. Finally, we give a brief conclusion in Section 4.

\section{Model and Theory}

The open mesoscopic structure will be described by a lattice Hamiltonian which generally reads as $H(t)=H_{\text {system }}+H_{\text {leads }}+\chi(t)\left(H_{\text {tun }}+H_{\text {int }}\right)$ where $H_{\text {system }}\left(H_{\text {leads }}\right)$ are the Hamiltonians of the disconnected subsystems (sample and leads) and the last time-dependent term contains the transfer Hamiltonian $H_{\text {tun }}$ accounting for the sample-lead coupling and the interaction part $H_{\text {int }}$. The Hamiltonian $H_{\text {system }}$ of the mesoscopic sample is be quite general and may describe a single quantum dot, double quantum dots (serially or parallel coupled) and other complex mesoscopic interferometers. The last two terms are introduced adiabatically through a smooth switching function $\chi(t)$. We shall denote by $d_{i}^{\dagger}\left(d_{i}\right)$ the creation (annihilation) operator associated to the $i$-th site of the lead. Similarly $a_{l}^{\dagger}\left(a_{l}\right)$ creates(annihilates) electrons in the $l$-th site of the sample. We write explicitly only the last two terms 
from the Hamiltonian:

$$
H_{\mathrm{tun}}=\sum_{i \in L_{\gamma}} \sum_{l \in C}\left(V_{i l}^{\gamma} d_{i}^{\dagger} a_{l}+h . c\right), \quad H_{\mathrm{int}}=\frac{U}{2} \sum_{l \neq m} \frac{\hat{n}_{l} \hat{n}_{m}}{\left|r_{l}-r_{m}\right|} .
$$

The notation $V_{i l}^{\gamma}$ stands for the hopping constant between the sites of the lead $\gamma$ and the ones in the sample. For simplicity a nearest neighbor form is taken so the only non-vanishing coupling will imply the endpoint site of the lead and the nearest site of the sample. $U$ is the interaction strength In the steady-state regime the current flowing from the lead $\alpha$ towards the sample is given in terms of the retarded and lesser Green functions through the well-known formula (see Ref. 2):

$$
J_{\alpha}=\frac{i e}{h} \int_{-2 t_{L}}^{2 t_{L}} d E \operatorname{Tr}\left\{\Gamma_{\alpha}\left(G^{R}-G^{A}\right) f_{\alpha}+G^{<}\right\}, \quad \Gamma_{l m}^{\alpha}(E)=2 \pi V_{i l}^{\alpha} V_{j m}^{\alpha} \rho(E) .
$$

The two Green functions should be computed from the Dyson and Keldysh equations i.e., $G^{R}=G_{0}^{R}+G_{0}^{R}\left(\Sigma_{L}^{R}+\Sigma_{I}^{R}\right) G^{R}$, and $G^{<}=G^{R}\left(\Sigma_{L}^{<}+\Sigma_{I}^{<}\right) G^{A}$, provided the retarded and lesser interaction self-energies $\Sigma_{I}^{R,<}$ are known. We have denoted by $G_{0}$ the Green function of the isolated and noninteracting central region. Also, $\Sigma_{L}$ stands for the leads' self-energy and has an analytical expression (see e.g. Ref. 8). $\rho(E)$ is the density of states at the endpoint of the semiinfinite lead and $f_{\alpha}(E)$ is the Fermi function of the lead $\alpha$. The bias across the system is introduced as the difference between the chemical potentials of the leads. In the case of two leads (Left and Right) we denote the bias by $V=\mu_{L}-\mu_{R}$. A more useful formula is the following (see Ref. 8 for a derivation):

$$
J_{\alpha}=\frac{e}{h} \int_{-2 t_{L}}^{2 t_{L}} d E \operatorname{Tr}\left\{\Gamma^{\alpha} G^{R} \Gamma^{\beta} G^{A}\left(f_{\alpha}-f_{\beta}\right)-\Gamma^{\alpha} G^{R} \operatorname{Im}\left(\Sigma_{I}^{<}+2 f_{\alpha} \Sigma_{I}^{R}\right) G^{A}\right\} .
$$

The scheme that we propose for the calculation of the interacting quantities is similar to the one employed by Faleev and Stockman ${ }^{10}$ in the study of elementary excitations in 2DEG, the difference being that we have to treat here an open system. Moreover, as the system has no translational invariance we will not perform the Fourier transform with respect to the position coordinates. We start from the contour ordered polarization operator which in the energy space has retarded and lesser components $(k, l$ denote sites from the central region only):

$$
\begin{aligned}
\Pi_{k l}^{<,>}(E) & =-\frac{1}{2 i} \int d E^{\prime} G_{k l}^{<,>}\left(E^{\prime}\right) G_{l k}^{>,<}\left(E^{\prime}-E\right) \\
\Pi^{R}(E) & =\frac{i}{2 \pi} \int d E^{\prime} \frac{\Pi^{>}\left(E^{\prime}\right)-\Pi^{<}\left(E^{\prime}\right)}{E-E^{\prime}+i 0} .
\end{aligned}
$$

Notice that the Green functions from the above equations are yet to be determined. The first approximation (sometimes called the $G_{0} W$ method) implies the change $G \rightarrow G_{\text {eff }}$ where $G_{\text {eff }}$ is the Green function of the non-interacting but coupled system. More precisely, $G_{\text {eff }}=G_{0}+G_{0} \Sigma_{L} G_{\text {eff }}$ being $G_{0}$ the Green function of the isolated sample. Since $G_{\text {eff }}$ is known the polarization operator can be computed. 
If it is then used to compute the remaining interacting quantities one gets a nonselfconsistent scheme which is nevertheless useful at least in the low bias regime. The interaction self-energy acquires a compact form:

$$
\Sigma_{I}=\Sigma_{H}+\Sigma_{X}+\Sigma_{C}
$$

where the Hartree, exchange and correlation contributions can be identified easily ${ }^{11}$ In a more elaborate approach one should use the above ansatz just as a zeroth order step in a selfconsistent scheme. This is the approach we have taken here.

The selfconsistent procedure that we have implemented here is more complex than the one used in Ref. 4 and could pose some problems when applied to bigger systems. This is because at every iteration step one has to perform double integrals over energy which are not trivial as they contain Green functions with sharp peaks. The number of peaks increases with the number of sites and moreover, they get sharper if the coupling to the leads is small. Then the energy grid should be carefully chosen such that all the peaks are properly resolved. Below, we have considered a 6-site quantum dot for which 1500 energy steps were required.

\section{Results and Discussion}

We now present a few illustrative examples underlying the Coulomb effects and the self-consistent procedure we have set out.

We first consider a $3 \times 2$-site quantum dot and calculate the current passing through it. The hopping constant between the leads and the dot will be denoted by $\tau$. In Fig. 1(a) one notices the six Coulomb peaks for the interacting and noninteracting case while in Fig. 1(b) we compare the two contributions in the current formula Eq. (3). The first contribution gives essentially the peak structure and it actually counts the poles of the retarded Green functions. When the gate potential is such that one of the renormalized QD levels is brought into the bias window the real part in the denominator $E-E_{n}-\Sigma_{L}^{R}-\Sigma_{I}^{R}$ of the Green function vanishes for some value of $E$. Consequently, a peak appears in the current.

The second term prevents one from casting the current in a Landauer form and contains the correlation effects. This is because a non-vanishing imaginary part of the self-energy starts can be obtained only if we compute at least the second order contribution with respect to the interaction strength. The contribution of this term turns out to be relevant for the first 3-4 peaks. We remark that in the case of the first (leftmost) peak the correction to the Landauer formula gives actually half of its amplitude. This peak corresponds to the tunneling out of one electron from the highest level of the QD. There are still 5 electrons within the dot so that the interaction effects turn out to be strong even for $U=0.15$.

We turn our attention now to a 3-site quantum dot and look at the so called Coulomb diamonds which are actually $3 \mathrm{D}$ plots of the current or the conductance as a function of the plunger gate voltage and of the applied bias. Usually these plots are obtained by imposing some relations between the chemical potential of the leads 

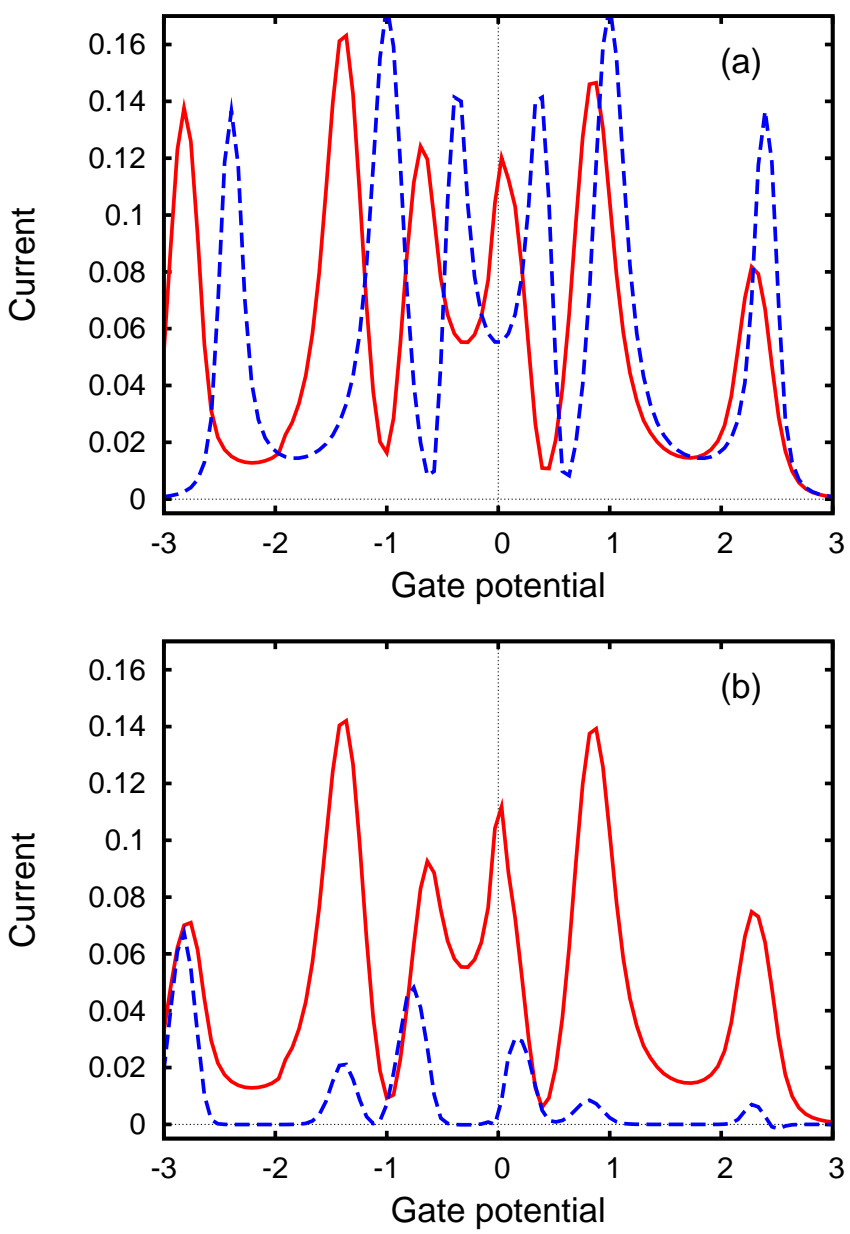

Fig. 1. (Color on line) (a) The steady state current for $U=0.15$ (solid line) and $U=0$ (dashed line). The interaction shifts the peaks to the left and broadens them. Note that the Hartree shift decreases as the quantum dot empties. (b) The separate contributions of the two terms in Eq. (3). Solid line (first term), dashed line (second term). Other parameters are $\tau=0.5, V=0.2$, $k T=10^{-4}$.

and of the dot ${ }^{1}$ and very few calculations based on a quantum transport formalism are available. In the present approach we are able to compare the interacting and non-interacting Coulomb diamonds for the steady-state current. The bias is varied as follows: we start by taking $\mu_{L}=-1$ and $\mu_{R}=1$, that is we start with a negative bias and the current will flow from the right lead to the left one. For the clarity of the figure we choose to represent the absolute value of the current. Then we increase/decrease the chemical potentials i.e $\mu_{L} \rightarrow \mu_{L}+\delta / 2$ and $\mu_{R} \rightarrow \mu_{R}-\delta / 2$ until we reach the situation $\mu_{L}=1$ and $\mu_{R}=-1$. 
(a)

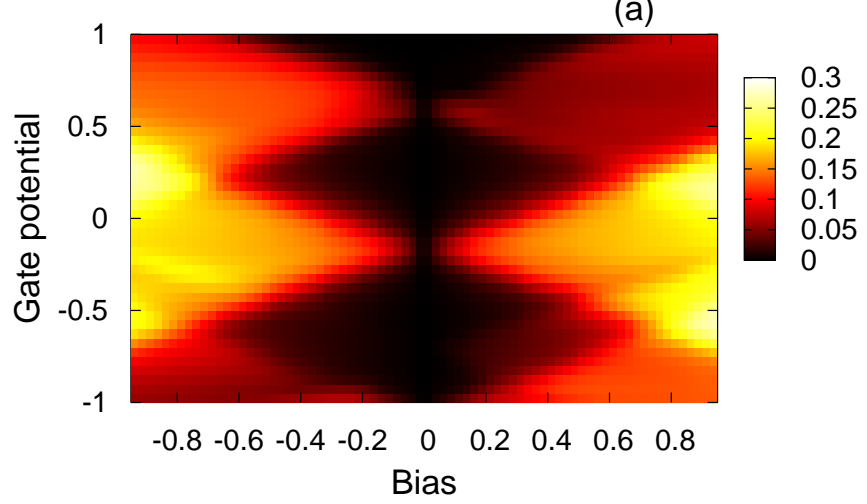

(b)

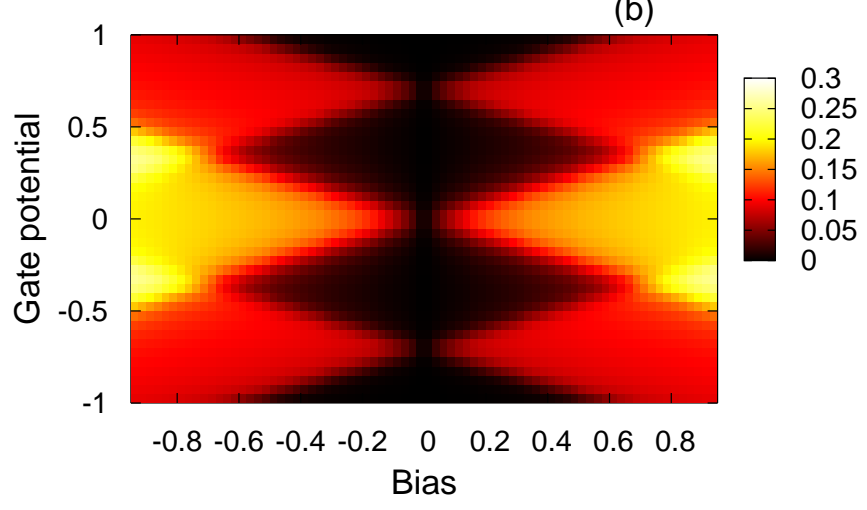

Fig. 2. (Color on line) Interacting (a) and non-interacting (b) self-consistent Coulomb diamonds for a 3-site dot. The interaction strength is set to $U=0.15$ and the bias is $V=0.2$. Other parameters are $\tau=0.5, k T=10^{-4}$.

In Figs. 2(a) and (b) we show the Coulomb diamonds in the interacting and non-interacting case. The dark regions correspond to the Coulomb blockade regime, where no level of the dot lies within the bias window. In this situation the number of electrons in the dot is a well defined integer. The crossing points around $V=0$ (also called vertex points) correspond to tunneling processes, as the gate potential is such that one level of the dot enters the bias window. We note that in the interacting case the Coulomb diamonds are not symmetric with respect to the value $V_{g}=0$ and that they are shifted toward lower values of $V_{g}$. This happens because the Coulomb interaction pushes the quantum dot levels upwards and then they could enter the bias window at even lower values of the gate potential. We note also that 

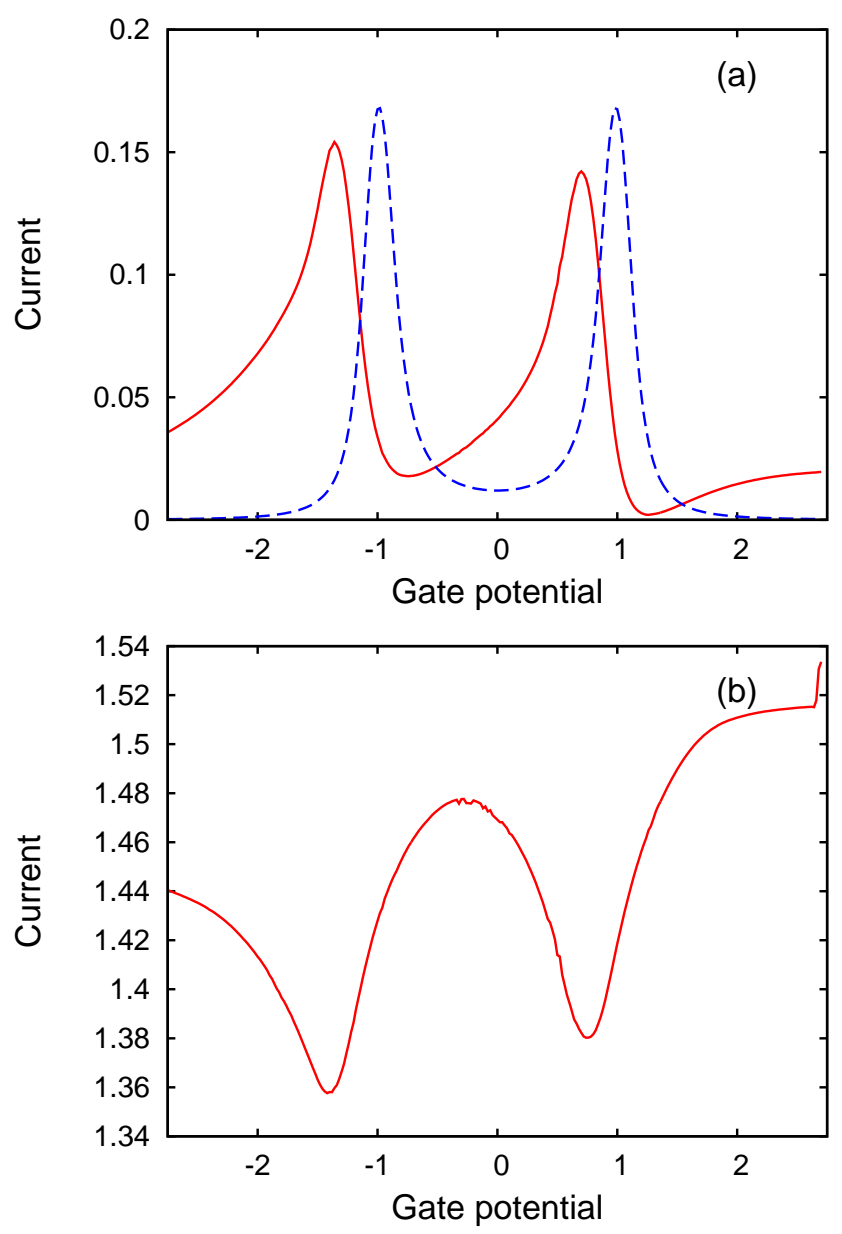

Fig. 3. (Color on line) (a) The current through $\mathrm{QD}_{1}$ as a function of the gate potential $V_{g}$ for $U=0.25$ (solid line) and $U=0$ (dashed line). (b) The current through $\mathrm{QD}_{1}$ exhibits dips that correspond to the resonant peaks in Fig.2(a). Other parameters are $\tau_{1}=0.35, \tau_{2}=1, k T=10^{-4}$.

in the presence of the interaction the 'length' of the diamonds is not the same as in the noninteracting case.

As a last application of the RPA-Keldysh method we have considered two parallel quantum dots in a four lead geometry, that is, each dot is coupled to two leads and therefore can be individually biased. In this case the coupling to the leads are denoted by $\tau_{1}$ and $\tau_{2}$. In order to include both the interdot and the intradot interactions one needs at leads two levels in each dot. A plunger gate potential applied on $\mathrm{QD}_{1}$ tunes it on resonance and the steady-state current exhibits two peaks (see Fig. 3(a)). The similar current through $\mathrm{QD}_{2}$ shows two dips that correspond to the tunneling processes in $\mathrm{QD}_{1}$. Evidently this behavior is entirely due to the 
interdot interaction. We stress that an accurate correspondence between the dips and the peaks in Figs. 3(a),(b) is obtained only if we perform the selfconsistent calculation. In the $\mathrm{G}_{0} \mathrm{~W}$ approach the polarization operator is constructed with the non-interacting Green functions whose pole structure misses the Hartree shift.

\section{Conclusion}

We have studied several interaction effects in open quantum dot systems in the non-linear transport regime within a selfconsistent random-phase approximation. The method relies upon the Keldysh Greens' function formalism and reproduces qualitatively the Coulomb diamond structure for a many-level quantum dot, as well as the charge sensing effect for a parallel quantum dot in a four lead geometry. The method goes beyond the usual selfconsistent mean-field approach and treats on equal footing the intradot and interdot Coulomb interaction. Further developments should include the spin-degree of freedom which was neglected in the present work.

\section{Acknowledgments}

B. T. is supported by TUBITAK (No. 106T052) and TUBA. V. M. acknowledges the hospitality at the Bilkent University where this work was done.

\section{References}

1. W. G. van der Wiel, S. De Franceschi, J. M. Elzerman, T. Fujisawa, S. Tarucha, and L. P. Kouwenhoven, Rev. Mod. Phys. 75, 1 (2002).

2. H. Haug and A.-P. Jauho, Quantum Kinetics in Transport and Optics of Semiconductors (Springer, Berlin, 1996).

3. V. Kashcheyevs, A. Aharony, and O. Entin-Wohlman, Phys. Rev. B 73, 125338 (2006).

4. L. E. Henrickson, A. J. Glick, G. W. Bryant, and D. F. Barbe, Phys. Rev. B 50, 4482 (1994).

5. E. Buks, R. Schuster, M. Heiblum, D. Mahalu, and V. Umansky, Nature (London) 391, 871 (1998).

6. A. Silva and S. Levit, Phys. Rev. B 63, 201309(R) (2001).

7. V. Moldoveanu, M. Ţolea, and B. Tanatar, Phys. Rev. B 75, 045309 (2007).

8. V. Moldoveanu and B. Tanatar, Phys. Rev. B 77, 195302 (2008).

9. K. S. Thygesen and A. Rubio, Phys. Rev. B 77, 115333 (2008).

10. S. V. Faleev and M. I. Stockman, Phys. Rev. B 66, 085318 (2002); 62, 16707 (2000).

11. The explicit expression can be traced from Ref. 8: $\Sigma_{C}$ is given in Eqs. (21)-(24), while the exchange and Hartree contributions are given by Eqs. (25) and (26). 